\title{
Revascularización coronaria sin circulación extracorpórea, experiencia quirúrgica en la Clínica de Especialidades Latino: serie de casos
}

Coronary revascularization without cardiopulmonary bypass, surgical experience at the Latino Specialty Clinic: case series

Mauricio Wilfrido Trelles Correa. ${ }^{1}$, Edisson Martin Luna Castro. ${ }^{2}$, Hanny Estefania Yamunaque Vire. $^{3}$

\begin{abstract}
DOI: https://doi.org/10.33262/cienciadigital.v3i4.2.1021

Introduction: Off-pump coronary artery bypass surgery (OPCAB), is a safe, cost-effective and reproducible surgery. Technique that has revolutionized coronary access and reduces the adverse effects of extracorporeal circulation. Method: A retrospective, descriptive study of four patients who underwent coronary revascularization without a pump at the Specialty Clinical Latino, in the City of Cuenca between 2016 - 2017. Results: The group consisted of a patient with an average age of 67.5 years, male; $50 \%$ with lesion of 3 vessels, an average LVEF of $40 \%$ and mortality between 4 and $8 \%$. The use of an internal mammary implant was necessary in all cases, and 50\% of the cases were also saphenous vein. An average of 2.75 units of red blood cell concentrates was necessary during the hospital stay. In the immediate postsurgical control, an average decrease of 81,000 / ul platelets was evidenced. Upon hospital discharge, the patients had a glomerular filtration rate of $83.76 \mathrm{ml} / \mathrm{min} / 1.73 \mathrm{~m} 2$. There were no deaths in the short term. Conclusion: At six months of follow-up, patients undergoing coronary revascularization without extracorporeal circulation had good results with complete revascularization and graft permeability.
\end{abstract}

Keywords: Coronary artery, coronary artery bypass, Off-pump surgery

\section{Resumen}

Introducción. La cirugía de revascularización coronaria sin circulación extracorpórea (CEC) es una cirugía segura, rentable y reproducible Técnica que ha revolucionado el acceso de las

\footnotetext{
${ }^{1}$ Clínica de Especialidades Latino, matc2202@hotmail.com

${ }^{2}$ Clínica de Especialidades Latino, lunitamartin11@gmail.com

${ }^{3}$ Clínica de Especialidades Latino, hannbad@gmail.com
} 
coronarias y que disminuye los efectos adversos de la circulación extracorpórea. Método. Estudio descriptivo retrospectivo de cuatro pacientes en los que se realizó revascularización coronaria sin bomba en la Clínica de Especialidades Latino, de la Ciudad de Cuenca entre el año 2016 - 2017. Resultados. El grupo estuvo conformado por paciente con una edad promedio de 67.5 años, de sexo masculino; $50 \%$ con lesión de 3 vasos, una FEVI promedio de $40 \%$ y una mortalidad entre 4 y $8 \%$. Fue necesario en todos los casos la utilización de implante de mamaria interna y el $50 \%$ de los casos se utilizó también vena safena- Fue necesario un promedio de 2.75 unidades de concentrados de glóbulos rojos durante la estadía hospitalaria. En el control postquirúrgico inmediato se evidencio un descenso promedio de 81000/ul plaquetas. Al egreso hospitalario los pacientes tuvieron un filtrado glomerular sobre $83,76 \mathrm{ml} / \mathrm{min} / 1.73 \mathrm{~m} 2$. No se existieron defunciones a corta mediano plazo. Conclusión. A los seis meses de seguimiento, los pacientes sometidos a la revascularización coronaria sin circulación extracorpórea tuvieron buenos resultados con una revascularización completa y la permeabilidad del injerto.

Palabras Claves: arterias coronarias; Revascularización; cirugía sin circulación extracorpórea.

\section{Introducción}

La cardiopatía isquémica es una importante causa de morbilidad en nuestro medio, por lo tanto la revascularización coronaria es la cirugía de elección para el tratamiento de pacientes con enfermedad de tronco coronario izquierdo; enfermedad de tres vasos; y enfermedad de uno o dos vasos con compromiso proximal de la arteria descendente anterior (1).

Aunque durante los últimos 30 años se ha realizado cirugía cardiaca con circulación extracorpórea, hoy en día también se realiza cirugía de revascularización coronaria sin circulación extracorpórea con el fin de reducir las complicaciones postoperatorias asociadas con el uso de la misma, dentro de estas complicaciones tenemos: respuesta inflamatoria sistémica, disfunción cerebral, falla miocárdica, falla renal, inestabilidad hemodinámica, disfunción respiratoria, alteraciones de la coagulación y del sistema inmunitario (2). La cirugía cardiaca sin circulación extracorpórea ha demostrado además una reducción en la morbimortalidad quirúrgica.(3). En un estudio realizado por la sociedad española de cirugía torácica cardiovascular, fueron intervenidos 4949 pacientes por cardiopatía isquémica de los cuales 1560 fueron sin circulación extracorpórea, la mortalidad en la cirugía coronaria con circulación extracorpórea (CEC) fue del 3,15, y en la sin CEC, del 2,6\%.(4) evidenciándose que se reduce significativamente el riesgo de eventos cerebro vasculares (5). 
La cirugía coronaria sin CEC se considera una técnica alternativa de revascularización miocárdica y su uso ha aumentado progresivamente (6). Esta técnica se hace posible gracias a la creación y desarrollo de los estabilizadores epicárdicos que permiten la exposición e inmovilización de las arterias a revascularizar (7). Y cada vez más se van perfeccionando los diferentes dispositivos que permiten la realización de esta técnica.

Cualquiera de las técnicas que se utiliza en la revascularización coronaria tienen sus ventajas y desventajas sin embargo la cirugía sin la utilización de circulación extracorpórea muestra múltiples beneficios por ello el objetivo de esta publicación es el de describir la experiencia quirúrgica de cuatro casos de revascularización coronaria sin la utilización de circulación extracorpórea en nuestro medio.

\section{Método}

Estudio descriptivo retrospectivo de cuatro casos de pacientes con cardiopatía isquémica, previamente diagnosticados por cateterismo cardiaco. La recopilación de datos, el análisis y los informes fueron realizados en la Clínica de Especialidades Latino, entre el año 2016-2017. Se seleccionó todos los pacientes que fueron sometidos a revascularización coronaria sin circulación extracorpórea.

\section{Tratamiento}

En todos los pacientes se realizó una esternotomía media estándar. Se realizó revascularización de la arteria descendente anterior en los cuatro pacientes y se utilizó como parte de la técnica a la arteria mamaria interna izquierda, y en dos casos adicionalmente se requirió revascularización de la rama intermedia con la vena safena.

\section{Resultados}

Se recogió datos como experiencia de cirugía de revascularización coronaria sin el uso de circulación extracorpórea en 4 casos clínicos intervenidos en la clínica de especialidades latino entre el año 2016 y 2017; el grupo estuvo conformado por paciente con una edad promedio de 67.5 años con un máximo de 71 años; en cuanto al género fue conformado en su totalidad por pacientes masculinos. En lo que se refiere a antecedentes patológicos el 75\% de paciente tenía diagnóstico de hipertensión arterial, el 25\% diagnóstico de dislipidemia; además un paciente tuvo el antecedente de Tromboembolia Pulmonar hace 10 años y otro paciente diagnóstico de Ca de colon en remisión y revascularización miocárdica mediante cateterismo cardiaco hace 6 años. En cuanto 
a los hábitos lo más representativo fue que el $50 \%$ de pacientes eran fumadores con un índice plaquetas/año entre 10 a 20.

El tiempo de hospitalización tuvo una mediana de 14 días, aunque con valores muy variables por dispersión de datos. El diagnostico preoperatorio fue de 4 casos de cardiopatía isquémica, uno de ellos con angina inestable y otro paciente con disfunción ventricular severa. Se realizó el cálculo de la mortalidad mediante EUROSCORE II obteniéndose una mortalidad máxima de $18.42 \%$. El promedio de fracción de eyección del ventrículo izquierdo medida por ecocardiografía fue de $40 \%$ con un mínimo de $30 \%$, evidenciándose una mejoría al alta con una FEVI promedio de $48.7 \%$ con un mínimo de $35 \%$ y un máximo de $60 \%$.

El 50\% de los pacientes tuvo lesión de 3 vasos, el 25\% de 2 vasos y el otro $25 \%$ lesión de un vaso. En todos los casos el vaso con mayor afectación fue la descendente anterior. Fue necesario en todos los casos la utilización de implante de mamaria interna y el 50\% de los casos se utilizó también vena safena. Un paciente tuvo un procedimiento asociado durante el acto quirúrgico que fue la exceresis de mixoma de aurícula izquierda. El promedio de tiempo quirúrgico total fue de 225 minutos con un mínimo de 180 minutos y un máximo de 330 minutos.

Todos los pacientes fueron ingresados a la unidad de cuidados intensivos con un promedio de hospitalización de 7.5 días con un máximo de 12 días; en 3 de los casos el tiempo de ventilación mecánica máximo fue de 24 horas, pero en un caso fue necesario 8 días de ventilación mecánica. En el 100\% de los casos fue necesario ayuda vasopresora y en el 75\% uso de ayuda inotrópica. La mediana de perdida sanguínea durante el intraoperatorio fue de $500 \mathrm{ml}$ con un promedio de $575 \mathrm{ml}$, se utilizó el sistema de cellsaver en 2 de los casos con un promedio de recuperado de glóbulos rojos de $250 \mathrm{ml}$ y un máximo de $800 \mathrm{ml}$. Fue necesario un promedio de 2.75 unidades de concentrados de glóbulos rojos durante la estadía hospitalaria. Los pacientes ingresaron con una hemoglobina promedio de $13,8 \mathrm{gr} / \mathrm{dl}$ y hematocrito promedio de $41.2 \%$ con valores muy dispersos puesto que el hematocrito mínimo fue de 26,6\% y el máximo de 61,5\%. El control postquirúrgico inmediato obtuvo una hemoglobina promedio de $11,55 \mathrm{gr} / \mathrm{dl}$ y hematocrito promedio de $34,25 \%$. El promedio de hematocrito al alta fue de 39,52\% y el control de seguimiento a los 2-3meses fue de $39,72 \%$.

Se cuantifico el recuento plaquetario previo al procedimiento quirúrgico con un valor mínimo de 145000/ul y un máximo de 366000/ul, el control postquirúrgico inmediato evidencio un descenso promedio de $81000 /$ ul plaquetas, con valor mínimo de 98000/ul y un máximo de 25300/ul. Al alta 
la mínima de recuento de plaquetas fue de 180000/ul con un promedio de 29200/ul y el control en consulta externa a los 2-3meses fue sobre 280000/ul.

Figura 1

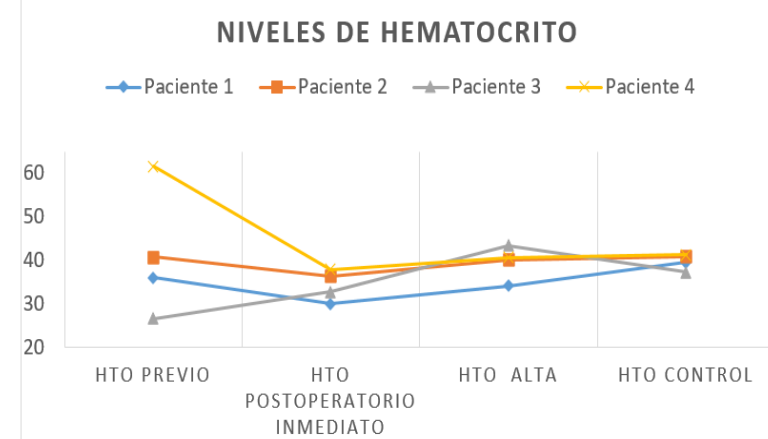

Figura 2

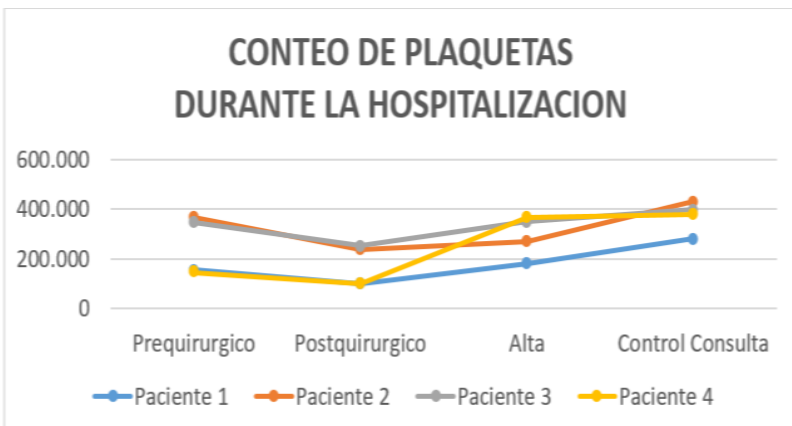

Fuente: Md Trelles, Md Yamunaque, Md Luna, Cuenca, Ecuador 2019

En cuanto a valores de azoados al ingreso los resultados obtenidos son una mediana de urea de $37,55 \mathrm{mg} / \mathrm{dl}$, con promedio de creatinina de $1,17 \mathrm{mg} / \mathrm{dl}$ y filtrado glomerular mínimo de $55.77 \mathrm{ml} / \mathrm{min} / 1.73 \mathrm{~m} 2$ según formula CKD-EPI. En el control postoperatorio inmediato se evidencio un valor promedio de urea de $38,15 \mathrm{mg} / \mathrm{dl}$, promedio de creatinina 1,22 y filtrado glomerular de $64,32 \mathrm{ml} / \mathrm{min} / 1.73 \mathrm{~m} 2$ con un mínimo de $37,79 \mathrm{ml} / \mathrm{min} / 1.73 \mathrm{~m} 2$. Al alta todos los pacientes tienen valores normales de azoados con un valor máximo de creatinina de $1,0 \mathrm{mg} / \mathrm{dl}$ y mínimo de filtrado glomerular de $83,76 \mathrm{ml} / \mathrm{min} / 1.73 \mathrm{~m} 2$.

Figura 3

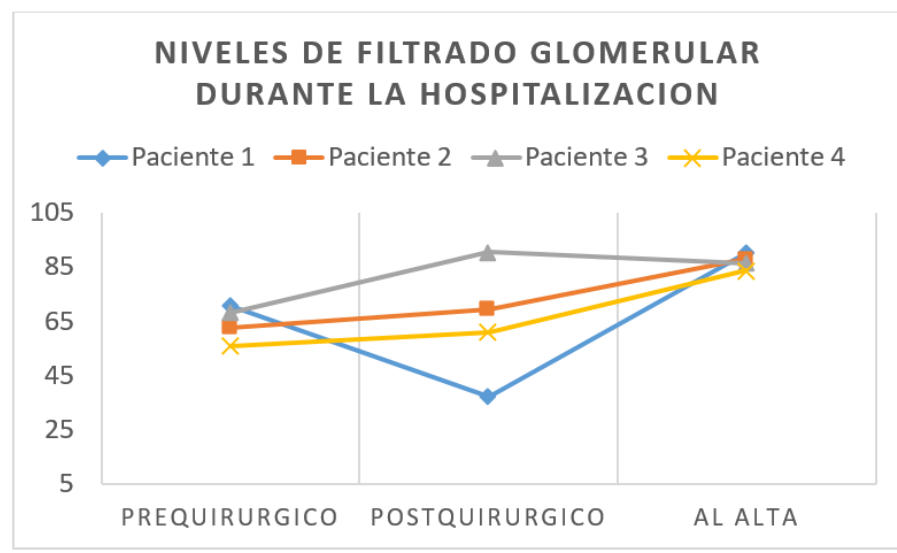

Fuente: Md Trelles, Md Yamunaque, Md Luna, Cuenca-Ecuador 2019

Dentro de las complicaciones durante la hospitalización, uno de los casos necesito al quinto día postoperatorio la colocación de un stent a nivel de arteria circunfleja y necesitó ventilación 
mecánica prolongada con necesidad de reintubación orotraqueal por choque cardiogénico secundario a nuevo proceso isquémico coronario, además un paciente tuvo insuficiencia renal aguda AKIN 1 que se resolvió previo al alta, No se reportó fallecimientos en esta serie de casos a corto y mediano plazo.

\section{Discusión}

El fundamento de este tipo de revascularización es evitar las complicaciones de la circulación extracorpórea, sobre todo el síndrome de respuesta inflamatoria sistémica y la anemia postoperatoria, de esta manera disminuir la morbimortalidad del paciente (8). Por lo tanto, la circulación extracorpórea aumenta los riesgos operativos, como el evento cerebrovascular, las lesiones miocárdicas, la insuficiencia renal aguda, el edema pulmonar, el sangrado y la infección (9). En un estudio de Kim HJ y sus colaboradores encontró la cirugía de recirculación coronaria sin CEC (OPCAB, siglas en inglés) se asoció con mejores tasas de supervivencia y preservación renal en comparación con la cirugía de revascularización coronario (CABG, por sus siglas en inglés) con CEC, sobre todo en pacientes con alto riesgo cardiaco (10).

Los cuatro casos descritos en este estudio fueron exclusivamente hombres con comorbilidades no cardiacas, pacientes con riesgo moderado - alto según la escala de euro-score, que se sometieron a cirugía de revascularización sin circulación extracorpórea. Numerosos estudios informan que la mortalidad por cirugía de revascularización coronaria es mayor en mujeres, por lo tanto la cirugía sin CEC puede tener un beneficio selectivo para las mujeres, reducir el infarto miocardio posoperatorio, pero no mejora significativamente otros resultados cardiovasculares, renales o neurológicos como describe Attaran y colaboradores (11). García Fuster et al. examinó a 547 pacientes consecutivos de un solo centro, de los cuales 121 tenían OPCAB, con un EuroSCORE más alto $(5,4 \pm 4)$ en comparación con el grupo $\operatorname{ONCAB}(2,8 \pm 2,3) \mathrm{y}$, a pesar de esto, no encontraron diferencias en la mortalidad y una tendencia en la reducción de la morbilidad y reducción de la transfusión ( $1 \pm 1$ vs. 1,9 \pm 2 unidades de sangre; $\mathrm{P}<0,0001$ ) y estadía hospitalaria postoperatoria $(8,9 \pm 5$ vs. $11,3 \pm 7$ días; $\mathrm{P}<0,001)$ a favor de cirugía sin CEC (12) .

La edad avanzada es un factor de riesgo conocido en la cirugía coronaria sin CEC (13), en el estudio presente la edad media es de 67.5 años, sin embargo, los resultados de un ensayo asignado al azar en pacientes ancianos mayor a 70 años que solicitaban cirugía de revascularización con CEC versus sin CEC, no mostró resultados significativos entre los dos grupos (14). Chowdhury et al. (15) informaron resultados favorables en pacientes con cirugía coronaria sin CEC, especialmente en 
aquellos con factores de alto riesgo, como edad avanzada, sexo femenino, disfunción del ventrículo izquierdo y antecedentes de accidente cerebrovascular o esternotomía previa.

El tiempo de ventilación mecánica asistida en la unidad de cuidados intensivos en el presente estudio fue de menos de 24 horas en tres de los cuatro pacientes, ya que solo uno de ellos requirió asistencia ventilatoria por 8 días, la ventilación prolongada se asocia con el tiempo de estadía hospitalaria (16). A demás en estos casos no hubo conversión de método durante la cirugía de revascularización intraoperatoria, el mismo se asocia con el aumento de la mortalidad, con un mayor riesgo de padecer casi todas las complicaciones perioperatorias que incluyen isquemia miocárdica, lesión, accidente cerebrovascular, insuficiencia renal y ventilación prolongada (17). Se requirió la ayuda de fármacos vasopresores e inotrópicos, el uso adecuado de estos fármacos ayuda a mejorar el gasto cardiaco y el tono vascular favoreciendo la clínica del paciente, en un estudio predijeron que la enfermedad coronaria izquierda y el uso de dos o más injertos fueron variables que se asociaron indirectamente con el uso intraoperatorio como posoperatorio de estos fármacos (18).

Los pacientes con FEVI baja no se consideran no aptos para cirugía revascularización sin CEC, en el estudio actual el porcentaje promedio fue de $40 \%$ y el mínimo fue de $30 \%$, en cuanto a la arteria más afectada fue la descendente anterior, algunos casos daño de 2 y 3 vasos, Li et al. [12] informaron que la enfermedad de la arteria coronaria principal izquierda, la insuficiencia cardíaca, la enfermedad coronaria de 3 vasos se asociaron con la conversión $(19,20)$.

La ausencia de cualquier daño miocárdico por isquemia o cardioplejía contribuyó a la recuperación de la función ventricular. Esto definitivamente abre una opción de tratamiento en pacientes con enfermedad coronaria crítica y disfunción grave del ventrículo izquierdo que generalmente se consideran inadecuados para cirugía coronaria sin CEC (21).

Una de las ventajas más importantes de la técnica revascularización coronaria sin circulación extracorpórea es que permite reducir la tasa de transfusiones de productos sanguíneos, según estudio el hematocrito se mantuvo más estable que en pacientes sometidos a cirugía convencional, probablemente debido a alteración en la cascada de coagulación (22). Se demostró en otro ensayo que relativamente la cirugía sin CEC existe menos activación de la coagulación y fibrinólisis y respuesta posoperatoria tardía en comparación que en el grupo con CEC (23). Además existe otros beneficios, todos los pacientes al alta tuvieron niveles normales de creatinina, con respecto a ello existe datos que estiman un riesgo mayor con el uso de CEC de lesión renal aguda $(24,25)$. 
En cuanto al seguimiento de los pacientes durante seis meses, el resultado fue satisfactorio, siendo la revascularización coronario completa con permeabilidad del injerto Los pacientes no presentaron deterioro cognitivo después de la cirugía. En otros estudios no se ha encontrado evidencia significativa en comparación con pacientes sometidos a cirugía coronaria con circulación extracorpórea (26). Sin embargo, los médicos que se oponen a la cirugía coronario sin CEC han insistido en que su dificultad técnica dificulta la revascularización completa, predispone a los injertos a una oclusión precoz, causa más evento cerebrovascular y produce resultados a largo plazo menos favorables. Además, pueden requerir circulación extracorpórea por varias razones, entre ellas, el paciente se encuentra en una condición inestable, disección difícil y anastomosis de las arterias coronarias diana, y revisión debido a un flujo inadecuado del injerto (9) .

\section{Conclusión}

La experiencia quirúrgica recolectada permite evidenciar que en nuestro medio es posible realizar revascularización miocárdica sin la utilización de circulación extracorpórea, permitiendo reducir las complicaciones del uso de CEC permitiendo al paciente ser dado de alta con valores normales de hto, plaquetas y función renal; además a los seis meses de seguimiento, los pacientes sometidos a la revascularización coronaria sin circulación extracorpórea tuvieron buenos resultados con una revascularización completa manteniendo la permeabilidad del injerto.

\section{Referencias Bibliográficas:}

1. Godinho AS, Alves AS, Pereira AJ, Pereira TS. On-pump versus off-pump coronary-artery bypass surgery: a meta-analysis. Arquivos Brasileiros de Cardiologia [Internet]. enero de 2012 [citado 11 de febrero de 2019];98(1):87-94. Disponible en: http://www.scielo.br/scielo.php?script=sci_abstract\&pid=S0066782X2012000100014\&lng=en\&nrm=iso\&tlng=en

2. Llorens León R. Cirugía coronaria sin bomba. Cir Cardiov [Internet]. 1 de enero de 2015 [citado 11 de febrero de 2019];22(1):10-1. Disponible en: http://www.elsevier.es/es-revistacirugia-cardiovascular-358-articulo-cirugia-coronaria-sin-bomba-S1134009615000169

3. Bustamante-Munguira J, Centella T, Polo L, Hornero. F. Cirugía cardiovascular en España en el año 2014. Registro de intervenciones de la Sociedad Española de Cirugía TorácicaCardiovascular. Cirugía Cardiovascular [Internet]. 1 de noviembre de 2015 [citado 11 de febrero de 2019];22(6):297-313. Disponible http://www.sciencedirect.com/science/article/pii/S1134009615002119

4. Cuenca JJ, Herrera JM, Rodríguez-Delgadillo MA, Paladini G, Campos V, Rodríguez F, et al. Revascularización miocárdica arterial completa con ambas arterias mamarias sin circulación extracorpórea. Rev Esp Cardiol [Internet]. 1 de mayo de 2000 [citado 11 de febrero de 2019];53(05):632-41. Disponible en: http://www.revespcardiol.org/es/revascularizacionmiocardica-arterial-completa-con/articulo/9727/ 
5. González L R, Seguel S E, Stockins L A, Campos M R, Neira S L, Alarcón C E. Cirugía Coronaria: Revascularización miocárdica sin circulación extracorpórea. Revista chilena de cirugía [Internet]. diciembre de 2009 [citado 11 de febrero de 2019];61(6):578-81. Disponible en: https://scielo.conicyt.cl/scielo.php?script=sci_abstract\&pid=S071840262009000600016\&lng=es\&nrm=iso\&tlng=es

6. Jaramillo JS, Jaramillo JC, Villegas A, Durán MA, Montoya M, González G, et al. Revascularización coronaria sin bomba. Revista Colombiana de Cardiología. 2003;10(4):5.

7. Oviedo López A, López Valenzuela P, Martínez Hernández R, Galván R, Solorio S, Hernández-González MA. La cirugía de revascularización por toracotomía izquierda sin circulación extracorpórea, es una alternativa viable en la re-operación de puentes coronarios. Archivos de cardiología de México [Internet]. septiembre de 2008 [citado 11 de febrero de 2019];78(3):299-304.

Disponible

en: http://www.scielo.org.mx/scielo.php?script=sci_abstract\&pid=S140599402008000300009\&lng=es\&nrm=iso\&tlng=es

8. Bonilla R, Sánchez Córdova JC, Guzmán Ovalle JE, Cabrera Escobar JA, Galindo J, Cruz Molina JR, et al. Revascularización coronaria sin circulación extracorpórea: Unidad de Cirugía Cardiovascular «UNICAR» Guatemala C.A. Revista Costarricense de Cardiología [Internet]. diciembre de 2002 [citado 28 de enero de 2019];4(3):11-3. Disponible en: http://www.scielo.sa.cr/scielo.php?script=sci_abstract\&pid=S140941422002000300003\&lng=en\&nrm=iso\&tlng=es

9. Lim J, Lee WY, Ra YJ, Jeong JH, Ko HH. Analysis of Risk Factors for Conversion from OffPump to On-Pump Coronary Artery Bypass Graft. Korean J Thorac Cardiovasc Surg [Internet]. febrero de 2017 [citado 12 de febrero de 2019];50(1):14-21. Disponible en: https://www.ncbi.nlm.nih.gov/pmc/articles/PMC5295478/

10. Kim HJ, Chung JE, Jung JS, Kim IS, Son HS. Current Status of Off-pump Coronary Artery Bypass Grafting in Patients with Multiple Coronary Artery Disease Compared with On-pump Coronary Artery Bypass Grafting: The Korean National Cohort Study. Thorac Cardiovasc Surg. 2018;66(6):470-6.

11. Attaran S, Harling L, Ashrafian H, Moscarelli M, Casula R, Anderson J, et al. Off-pump versus on-pump revascularization in females: a meta-analysis of observational studies. Perfusion [Internet]. 1 de septiembre de 2014 [citado 11 de febrero de 2019];29(5):385-96. Disponible en: https://doi.org/10.1177/0267659114525985

12. Fuster RG, Montero JA, Gil Ó, Hornero F, Cánovas SJ, Dalmau MJ, et al. Advantages of OffPump Coronary Bypass Surgery in High-Risk Patients. Rev Esp Cardiol [Internet]. 1 de abril de 2002 [citado 12 de febrero de 2019];55(04):383-90. Disponible en: http://www.revespcardiol.org/en/advantages-of-off-pump-coronarybypass/articulo/13032023/

13. Shahian DM, O'Brien SM, Filardo G, Ferraris VA, Haan CK, Rich JB, et al. The Society of Thoracic Surgeons 2008 cardiac surgery risk models: part 1--coronary artery bypass grafting surgery. Ann Thorac Surg. julio de 2009;88(1 Suppl):S2-22.

14. Houlind K, Kjeldsen BJ, Madsen SN, Rasmussen BS, Holme SJ, Nielsen PH, et al. On-pump versus off-pump coronary artery bypass surgery in elderly patients: results from the Danish on-pump versus off-pump randomization study. Circulation. 22 de mayo de 2012;125(20):2431-9.

15. Chowdhury R, White D, Kilgo P, Puskas JD, Thourani VH, Chen EP, et al. Risk Factors for Conversion to Cardiopulmonary Bypass During Off-Pump Coronary Artery Bypass Surgery. 
The Annals of Thoracic Surgery [Internet]. junio de 2012 [citado 12 de febrero de 2019];93(6):1936-42. Disponible en: https://linkinghub.elsevier.com/retrieve/pii/S0003497512004110

16. Khan H, Uzzaman M, Benedetto U, Butt S, Raja SG. On- or off-pump coronary artery bypass grafting for octogenarians: A meta-analysis of comparative studies involving 27,623 patients. Int J Surg. noviembre de 2017;47:42-51.

17. Keeling B, Thourani V, Aliawadi G, Kim S, Cyr D, Badhwar V, et al. Conversion From OffPump Coronary Artery Bypass Grafting to On-Pump Coronary Artery Bypass Grafting. Ann Thorac Surg. 2017;104(4):1267-74.

18. Chooriyil N, Palappallil DS. Peri-operative Inotrope Use in Off Pump Coronary Artery Bypass Graft Surgeries. Journal of clinical and diagnostic research [Internet]. 2017 [citado 12 de febrero de 2019]; Disponible en: http://jcdr.net/article_fulltext.asp?issn=0973709x \&year=2017\&volume $=11 \&$ issue $=12$ \&page $=$ PC16\&issn $=0973-709 x \& i d=10998$

19. Li Z, Amsterdam EA, Danielsen B, Hoegh H, Young JN, Armstrong EJ. Intraoperative conversion from off-pump to on-pump coronary artery bypass is associated with increased 30 day hospital readmission. Ann Thorac Surg. julio de 2014;98(1):16-22.

20. Jin R, Hiratzka LF, Grunkemeier GL, Krause A, Page US. Aborted off-pump coronary artery bypass patients have much worse outcomes than on-pump or successful off-pump patients. Circulation. 30 de agosto de 2005;112(9 Suppl):I332-337.

21. Saha KK, Pinto B, Arya M, Saha KK. Total arterial anaortic off-pump coronary artery bypass grafting in a patient with heart failure - Case report. Indian Heart Journal [Internet]. 1 de septiembre de 2016 [citado 8 de febrero de 2019];68(5):700-3. Disponible en: http://www.sciencedirect.com/science/article/pii/S0019483215009438

22. Walczak M, Urbanowicz TK, Tomczyk J, Camacho E, Ligowski M, Stefaniak S, et al. Transfusion of blood products in off-pump coronary artery bypass and conventional coronary artery revascularization. A prospective randomized study. Kardiochir Torakochirurgia Pol [Internet]. junio de 2014 [citado 16 de febrero de 2019];11(2):136-9. Disponible en: https://www.ncbi.nlm.nih.gov/pmc/articles/PMC4283870/

23. Roy S, Saha K, Mukherjee K, Dutta S, Mukhopadhyay D, Das I, et al. Activation of Coagulation and Fibrinolysis During Coronary Artery Bypass Grafting: A Comparison Between On-Pump and Off-Pump Techniques. Indian J Hematol Blood Transfus [Internet]. diciembre de 2014 [citado 16 de febrero de 2019];30(4):333-41. Disponible en: https://www.ncbi.nlm.nih.gov/pmc/articles/PMC4243405/

24. Seabra VF, Alobaidi S, Balk EM, Poon AH, Jaber BL. Off-Pump Coronary Artery Bypass Surgery and Acute Kidney Injury: A Meta-Analysis of Randomized Controlled Trials. CJASN [Internet]. 1 de octubre de 2010 [citado 16 de febrero de 2019];5(10):1734-44. Disponible en: https://cjasn.asnjournals.org/content/5/10/1734

25. Gaipov A, Molnar MZ, Potukuchi PK, Sumida K, Szabo Z, Akbilgic O, et al. Acute kidney injury following coronary revascularization procedures in patients with advanced CKD. Nephrol Dial Transplant [Internet]. julio de 2018 [citado 16 de febrero de 2019]; Disponible en: https://academic.oup.com/ndt/advance-article/doi/10.1093/ndt/gfy178/5049870

26. Shroyer AL, Grover FL, Hattler B, Collins JF, McDonald GO, Kozora E, et al. On-Pump versus Off-Pump Coronary-Artery Bypass Surgery. New England Journal of Medicine [Internet]. 5 de noviembre de 2009 [citado 9 de febrero de 2019];361(19):1827-37. Disponible en: https://doi.org/10.1056/NEJMoa0902905 


\section{PARA CITAR EL ARTÍCULO INDEXADO.}

Trelles Correa, M., Luna Castro, E., \& Yamunaque, H. (2019). Revascularización coronaria sin circulación extracorpórea, experiencia quirúrgica en la Clínica de Especialidades Latino: serie de casos. Ciencia Digital, 3(4.2), 168-178. https://doi.org/10.33262/cienciadigital.v3i4.2.1021

\section{Liencia}

El artículo que se publica es de exclusiva responsabilidad de los autores y no necesariamente reflejan el pensamiento de la Revista Ciencia Digital.

El artículo queda en propiedad de la revista y, por tanto, su publicación parcial y/o total en otro medio tiene que ser autorizado por el director de la Revista Ciencia Digital.
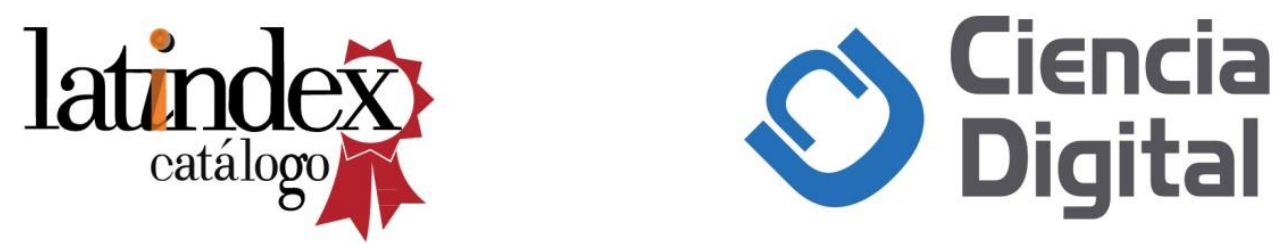